\title{
Application of Virtual Simulation Technology in Electronic Technology Industry
}

\author{
Qing Zhao
}

College of Electrical Engineering, Chongqing Institute of Engineering Chongqing, 400037, China

Keywords: electronic technology, virtual simulation technology, characteristics, application

\begin{abstract}
As the upgrading speed of product design and production is very fast and the market demands are increasingly growing with the economic development, the use of virtual simulation technology can improve the upgrading of electronic products, to promote the technical innovation of electronic industry. This paper mainly discusses the characteristics and application effects of virtual simulation technology in electronic technology industry, in the expectation of providing some theoretical references for the relevant practice.
\end{abstract}

\section{Introduction}

At the present stage, both the development scale and speed of electronic technology industry have changed a lot, huge capacity and quick development speed are the distinguishing features of today's electronic technology industry, and the electronic information products are closely related to the people's daily production and life. With the fast-changing science and technology, people have higher and higher demands for electronic products. To satisfy the diversified consumption and use demands, it is the only way of industry development to research and development electronic products with virtual simulation technology.

\section{Characteristics of Virtual Simulation Technology}

Virtual simulation technology, which is also known as virtual reality technology or simulation technology, is a technology to simulate the real system with virtual system depending on the computer technology. The emerging of virtual simulation technology can be traced back to the 1940s. It has gradually developed into a kind of general technology which applies to different industries, and it is of certain strategy, so it is an important method and means to carry out experimental study. In addition, with the economic development, the people have higher and higher requirements for virtual simulation technology, therefore making it from the traditional form to simulate the simple technology systems evolve to the high-end form to simulate complicated systems. In this process, the virtual simulation technology has combined with the technical development results in other fields, and so it has gradually realized the perfection.

The virtual reality technology has very strong integrity and integration characteristics in itself, and it is closely linked to many fields, such as computer graphics, human-computer interaction, satellite remote sensing technology, and AIT (artificial intelligence technology). Through the software and hardware of computer, and together with different sensors, a 3D simulation environment is formed, to represent the people and objects in the reality clearly, so that the users can get a realistic use effect and the human-computer interaction level is greatly improved. Based on the virtual reality technology, virtual simulation technology is integrated with the advantages of computer network communication technology, multimedia technology and etc., therefore forming a more powerful simulation technology, which has more obvious effect to represent the reality realistically. In the practical application, virtual simulation technology focuses on the systematic construction of virtual environment. In addition to grasping the overall effect of virtual environment from a macroscopic view, it pays attention to the construction at micro level, uniformly controlling and deploying the various entities by use of systematized virtual environment, so the operation is very easy and convenient. These control entities mainly include different simulators and also other virtual simulation subsystems, sometimes, a simple model can be used to represent the entities, so it is relatively flexible. Various entities in the virtual simulation environment all have different links; they can interact mutually and also have interaction with macro-environment. In this way, the characteristics of objective world are represented more clearly, with a quite high reality. As for the application of modern simulation technology, the integration characteristics in virtual technology shall be fully blended, and meanwhile, it is required to take advantage of its technical characteristics 
of networking and virtualization.

With virtual simulation technology, the customers can fully mobilize various sense organs including vision, audition and touch to get the information resources in virtual environment. In a simulation system, only by comprehensively considering different sensory effects, can be an ideal effect of virtual simulation technology application achieved.

In practical application, the characteristics of virtual simulation technology are mainly reflected at four aspects, namely, immersion, interaction, illusoriness and reality. These characteristics are organically integrated in an integral whole, and they depend on each other. Interaction is a main characteristic in the operation process of virtual simulation technology, this is mainly because of the manual control and management of virtual environment, and such manual control has a spontaneous characteristic, which is the control is realized through human's body language, so virtual simulation environment is adaptable according to the people's demands, and the initiative and flexibility of system operation can be improved. Although virtual simulation environment is visional, it can represent the real environment to the life, and moreover, it can simulate the things that are nonexistent in the reality, not only making people have immersive feeling but also enriching the people's imagination to the largest degree, to provide more possibilities for the industrial development.

The development of electronic technology industry is increasingly depending on the application advantage of virtual simulation technology, more importantly, the problems in design and production of electronic products can be solved by use of virtual simulation technology. For example, if virtual simulation technology is adopted in the inspection and analysis process of mechanical property of electronic products, it is able to present the use reliability of electronic products intuitively, predict their service life, and establish a reasonable maintenance and early-warning mechanism. In addition, the impact resistance of electronic products, the carrier vibration effect, and the possible problems in the adapter design process can be solved through virtual simulation technology. As for the thermal performance analysis of electronic products, the research of chip packaging and welding spot fatigue, and the measurement of indexes like vibration noise, virtual simulation technology will also be used. In electronic industry, MSC.Software Company has produced lots of virtual simulation software, therefore providing more convenience to promote the structural improvement and upgrading of electronic products. For example, with MSC.ADAMS, MSC.Marc, and MSC.Dytran, the research of various performances and functional development of electronic products are integrated together, and also the mutual integration of electronic technology industry and other subjects has been promoted, therefore, the development of virtual simulation technology is more promising.

\section{Application of Virtual Simulation Technology in Electronic Technology Industry}

In the current development process of electronic technology industry, there are no uniform virtual technology specifications, so various technical problems can only be solved according to practical needs and operation experience, and the operation efficiency shall be improved. With virtual simulation technology, we can not only solve the technical problems in the industry effectively but also analyze the problems in cross fields of many industries scientifically, making a corresponding joint simulation modeling analysis according to the coupling degree and characteristics of problems.

The application of virtual simulation technology software in electronic technology industry is mainly reflected in the following aspects:

First, the application in the analysis process of electronic industry structure, with MSC.Nastran as an example. In the analysis of industry structure, MSC.Nastran is the first choice for use. Such kind of virtual technology simulation software has very powerful analysis ability in static force, buckling, advanced dynamic response, random vibration response, and etc. With it, it is able to comprehensively analyze and understand the design sensitivity, structure optimization characteristics and practice measures of electronic products, therefore providing scientific foundations for the development of electronic technology industry. Such technology software has a very reliable operability. It is proved by many tests and engineering practice that this technology 
software has a good application effect, and it is widely applied by world-famous electronic and electrical product enterprises.

Second, an obvious effect of application in nonlinear analysis, thermal performance analysis, and electromagnetic performance analysis of electronic technology industry. The virtual simulation technology can provide various technical application systems for the nonlinear analysis of electronic industry, including the nonlinear analysis of materials, geometry and contact characteristics, etc. The most widely used software is MSC.Marc. It is the first software in the world to establish nonlinear finite element analysis system, which not only has a powerful nonlinear analysis function but also can meet the analysis demands of thermal performance, flow field characteristics, magnetic field characteristics, electric field, sound field and other aspects, and moreover, it can make a macro-micro comprehensive analysis of the coupling performance of multi-physics field, which facilitates to comprehensively understand the production characteristics and application features of electronic products. With this virtual simulation technology, it is able to make process and technology simulation to the processing and production of electronic products and to improve the control precision of the whole process, so as to increase the working efficiency. This software plays an important role in judgment of crack expansion degree, calculation of shock resistance, and analysis of coupling performance. According to the analysis on the thermal balance effect generated and the heat dissipation demands in the use of electronic products, the thermal effect of electronic products can be analyzed and recorded in all directions by use of the virtual simulation technology software, with the main content including thermal conduction effect, convection effect, radiant efficiency, contact heat transfer efficiency, etc., and moreover, double analysis of steady and dynamic states for gap heat transfer of electronic products can be made, therefore greatly facilitating the design and production of electronic products, and meanwhile, increasing the standard and reliability of electronic technology industry.

Third, the application in analyzing the drop and collision of electronic products. For example, virtual simulation technology can be used to simulate the characteristics in drop and collision of mobile phones, so as to provide a reliable data support for the drop design of electronic products. The most commonly used software is MSC.Marc and LS-DYNA. In the practical application, such software integrates their respective advantages, and their typical feature is to provide highly nonlinear transient dynamics analysis function with a higher precision. In the process of analyzing the drop process of electronic products, the characteristics of product drop can be analyzed according to the characteristics of electronic products, and moreover, based on these characteristics, corresponding measures will be adopted to reduce the loss after drop and collision, to effectively prolong the service life of electronic technology products and also provide more conveniences for customers.

Fourth, in the control and utilization of the motion simulation of electronic products and the motion system of electronic technology, virtual simulation technology plays a key role. The most common software includes MSC.ADAMS software and MSC.EASY5 software. With the software, a simulation study can be made for the mechanical property of electronic products, to get relatively accurate dynamic simulation data index and to provide necessary data for the production of electronic products. For example, through the dynamic virtual simulation research of robots, the various performances of robots in actual use can be judged, and it can be used for the simulation for automatic system operation motion of electric switch. In the research of radar antenna, it is able to more exactly judge the motion disciplines and the use effect of radar control system by use of MSC.ADAMS, and also MSC.ADAMS and MSC.EASY5 can be combined for joint simulation and control effect. With MSC.Nastran, it is able to more exactly calculate the wind load conditions of radar antenna system and judge the specific conditions of vibration load, to exactly analyze the static response and dynamic response, so that the radar antenna system users can grasp the use rules more conveniently and apply it to the practical production.

Fifth, the application in the calculation and analysis of fatigue in the electronic industry. The most common analysis software is MSC.Fatigue, and with this software, it is able to more exactly calculate the use fatigue and the final service life limit of electronic products, and a better effect can 
be got in the structural analysis of electronic products. Exactly grasping the thermal fatigue index of electronic products is helpful to understand the product performances more concretely and comprehensively, and reasonable use and maintenance strategies can be worked out based on characteristics, to facilitate to extend the product service life to the maximum degree. Such software is widely applied in the fatigue calculation of circuit board welding spots of electronic products, and an expected effect can be got.

Sixth, an obvious application effect in the noise analysis of electronic products. The most common analysis software is MSC.Actran, and such software is especially used for research of noise decibel. The principle of application includes finite element analysis and infinite element analysis. If the two are organically combined to be used, according to theory, it is able to exactly calculate the radiation, diffraction, scattering, oriented transmission, enclosed sound field, and absorption of sound as well as other characteristics in the use of electronic products, therefore realizing an exact modeling effect of sound. In the research of classical monopole-type sound and the analysis and research of higher-level physical model, through the combined use of the two, not only a relatively reasonable noise solution can be provided for electronic products, but also the application effect of internal loudspeaker can be optimized, so as to provide a more effective guarantee for the sound effect of electronic products. With the continuous development of electronic technology industry, more and more problems have occurred to this field, and the application of virtual simulation technology can provide diversified and personalized service for the production needs of electronic products, which shortens the cycle of upgrade of products and meanwhile reduces the errors caused by manual operation to the largest degree, therefore improving the application performance and effect of electronic products and promoting the sustainable and precision-oriented development of electronic industry.

\section{Summary}

In conclusion, the electronic technology industry in the modern society is featured by a very short product research \& development cycle and a higher frequency of product upgrading and replacement. To meet the diversified demands of different customers for electronic products maximally, an ideal effect can be got with the application of virtual simulation technology. It can not only promote the structural optimization and improvement of electronic products, but also push forward the continuous innovation in core technology, so as to lay a firm foundation of technology and method for the future development of electronic industry and further to develop a more broad space for the sustainable development of the industry.

\section{References}

[1] Li Songxiang. Research on Application of Virtual Simulation Technology in Electronic Technology Courses Teaching [D]. Hunan Normal University, 2012 (01)

[2] Lin Ruobo, Peng Yanbiao, Chen Bingwen, Fang Chuncheng. Application of Virtual Simulation Technology in "Electric and Electronic Technology" Courses Teaching [J]. Journal of Yunnan Nationalities University (natural science), 2013, 09 (10)

[3] Shi Kun. Exploration and Analysis of Computer Simulation Technology in Electronic Technology Courses [J]. Intelligence, 2012, 01 (15)

[4] Wang Lijing: Study of Virtual Simulation Technology in Electronic Experiments [J]. Electronics, 2013, 09 (15)

[5] Qiu Dongmei. Realization of Virtual Community Automatic Planning and Indoor Computer-Aided Design System [D]. Ocean University of China, 2013, 05 (10)

[6] Liu Yong. Application Value of Virtual Simulation Technology in Refrigeration Maintenance Teaching in Secondary Vocational Schools [J]. Education Information Technology, 2013, 08 (05)

[7] Cao Defang. Research and Development of Camshaft CNC Grinding Process Intelligent Application System [D]. Hunan University, 2012, 03 (15)

[8] Chen Wei, Lu Hongbin, et al. Research on Application of Virtual Simulation Technology in Product Development [J]. Development \& Innovation of Machinery \& Electrical Products, 2011 